\title{
Les Chironomidés (Diptera) du Liban. II. Description de l'imago ơ et de la nymphe de Thienemannia libanica n.sp.
}

H. Lavillel

Z. Moubayed

Mots-clès: Diptera, Chironomidae, Orthocladinae, nouvelle espèce, Liban.

Les auteurs décrivent l'imago ơ et la nymphe d'une nouvelle espèce récoltée dans la rivière Yammouné (Bekaa-Liban) -t la comparent avec la seule espece europeenne connue du genre Thienemannia gracilis $\mathrm{K}$.

The chironomids (Diptera) from Lebanon. II. Description of the male imago and pupa of Thienemannia libanica n. sp.

Keywords : Diptera, Chironomidae, Orthocladiinae, new species, Lebanon.

The authors describe the male imago and pupa of a new species, Thienemannia libanica, collected in the River Yammoune (Bekaa-Lebanon), and compare it with the only European species known from this genus, Thienemannia gracilis $\mathrm{K}$.

Lors de recherches sur la faune chironomidienne de quelques réseaux hydrographiques de la plaine de la Bekaa du Liban (Moubayed \& Laville 1983), nous avons trouvé $7 \%, 4 \mathrm{~N} \odot, 4 \mathrm{~N} \&$ d'une nouvelle espèce appartenant au genre Thienemannia jusqu'ici monospecifique (Th. gracilis K.). Cette espèce a été récoltée par dérive et en vol à Chlifa $\left(34^{\circ} 10^{\prime}\right.$ latitude $\mathrm{N}, 36^{\circ}$, longitude $\mathrm{E}$ ) dans la rivière Yammouné, petit torrent du versant Est du MontLiban.

L'imago ơ et la nymphe sont décrites dans ce travail suivant la terminologie générale de Saether (1980).

L'hypopyge $\sigma$ présente un gonostyle triangulaire caractéristique ; la nymphe se différencie de celle de $T h$ gracilis par l'absence d'ornementation au centre du $\mathrm{VI}^{\mathrm{e}}$ tergite abdominal.

1. Laboratoire d'Hydrobiologie, (U.A. 695 du C.N.R.S.), Université P. Sabatier, 118 route de Narbonne, 31062 Toulouse Cedex France.
Thienemannia libanica n. sp. (Fig. 1 à 3)

LOCALITE TYPE : Liban, torren: Yammouné, Chlifa (1 100-1 $200 \mathrm{~m}$ ).

MATÉRIEL TYPF:

- Holotype or Liban, Yammouné, Chlifa, 16.4.1982, collection Muséum Université libanaise (Beyrouth).

- Paratypes: 4 orêmes références que l'holotype: $2 \circ$, collection personnelle Laboratoire Hydrobiologie, Toulouse (France).

- Nymphes : $2 \mathrm{~N}$ o, $4 \mathrm{~N} Q$ montées, $2 \mathrm{~N}$ \& conservées dans l'alcool, collections Beyrouth et Toulouse.

DIAGNOSE:

- Imago $\sigma^{*} \mathrm{Ar}=0,25$, yeux pubescents, 15.17 soies acrosticales, aile avec quelques macrotriches à l'extrémité distale, squama avec 1.3 soies. Eperon du tibia antérieur : $18-19,5 \mu \mathrm{m}$ de long, éperons du tibia moyen $15 \mu \mathrm{m}$ et $12 \mu \mathrm{m}$ de long, éperons du tibia postérieur $12.15 \mu \mathrm{m}$ et $30 \mu \mathrm{m}$ de long. Hypopyge : pointe anale sans soies, gonostyle triangulaire. 


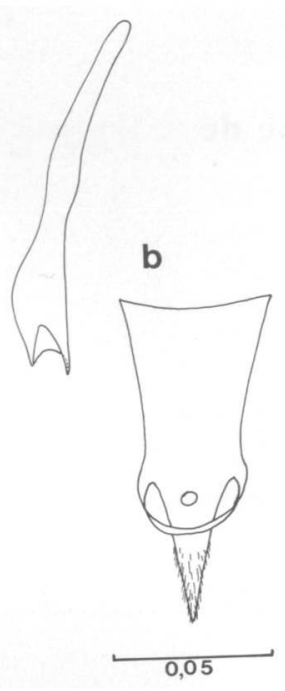

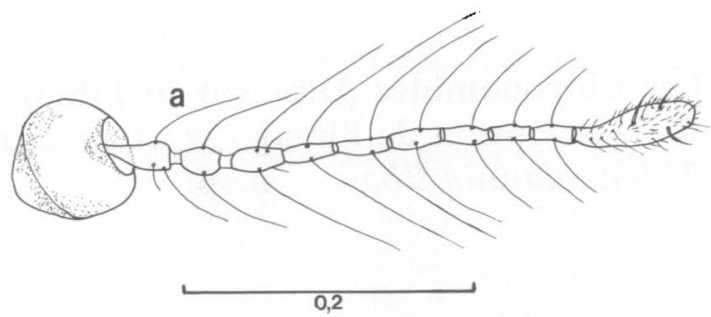

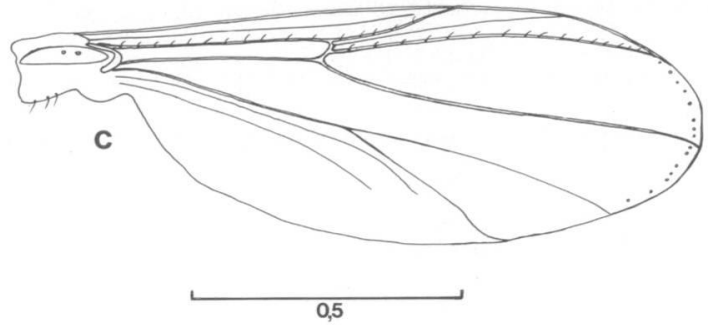

Fig. 1. Thienemannia libanica $\mathbf{n} . \mathbf{s p}, \sigma$ :

a, antenne - b, cibarial pump (palpe céphalique interne) et tentorium - c, aile. Echelle en mm.

- Nymphe: $\mathrm{L}=\mathbf{2}, 1-2,3 \mathrm{~mm}$. Pas de corne thoracique. Tergites VII et VIII avec une plaque médiane plus ou moins circulaire d'épines. Soies latérales des segments abdominaux courtes.

\section{DESCRIPTION :}

Mâle (n $=3$ )

Longueur totale : 1,3-1,4 mm.

Longueur de l'aile : $1,25 \mathrm{~mm}$; largeur : $0,45 \mathrm{~mm}$; VR $=1,25$.

Antenne (fig. $1 \mathrm{a}): 11$ articles ; Flagellomères L/1 (microns), 69/87, 39/21, 30/19,5, 36/13,5, 36/10.5, $36 / 10,5,36 / 9,33 / 9,30 / 9,27 / 10,5,78 / 24 . \mathrm{AR}=0,25-0,27$ (même AR que Th. gracilis); article 2 à 10 avec un seul verticille de soies longues de 60 à $160 \mu \mathrm{m}$; dernier article renflé distalement, avec de petites soies terminales recourbées.

On note que Brundin (1956) signale 12-13 segments antennaires chez Th. gracilis.

\section{- Tête}

Yeux pubescents, légèrement prolongés dorsalement, vertex avec $4-5$ soies derrière chaque ceil ; clypeus avec 7 soies alignées sur une rangée transversale; longueur des segments des palpes (palpomères 2-5) $33 \mu \mathrm{m}, 57 \mu \mathrm{m}, 75 \mathrm{~mm}, 75 \mu \mathrm{m}$.

Cibarial pump (palpe céphalique interne) et tentorium comme sur la figure $1 \mathrm{~b}$.

\section{- Thorax}

15 à 17 soies acrosticales (acrostichal setae) disposées sur 1 ou 2 rangées, 17-20 soies dorsocentrales (dorsolateral setae) sur 2 rangées, 4 soies préalaires, 6 soies sur une rangée au scutellum.

\section{- Aile (fig. $1 \mathrm{c}$ )}

Trois fois plus longue que large ; quelques macrotriches le long du bord distal : chez $T$. gracilis les macrotriches sont réparties sur toute la surface. VR $=1,25$. Lobe anal très réduit, squama avec $1-3$ soies, Brachiolum avec 2 soies, $R$ avec $9-11$ soies. $R I$ avec 
5 soies, R4 + 5 avec $\mathbf{1 5 - 1 7}$ soies, se terminant nettement au-delà de $\mathrm{Cu}_{1}$, Costa se prolongeant au-delà de $\mathrm{R} 4+5$, $\mathrm{fCu}$ légèrement distal de $\mathrm{Rm}, \mathrm{Cu}_{2}$ légèrement ondulée à son extrémité, An se terminant au niveau de Fcu.

\section{- Pattes ( $n=1)$}

Longueur des éperons des tibias : P1 18-19,5 $\mu \mathrm{m}$; P2 $15 \mu \mathrm{m}$ et $12 \mu \mathrm{m}$; P3 $15 \mu \mathrm{m}$ et $30 \mu \mathrm{m}$. Peigne ti3 avec $14-15$ soies. Largeur apex des tibias : P1 $27 \mu \mathrm{m}$; P2 24-25 $\mu \mathrm{m}$; P3 33-39 $\mu \mathrm{m}$. Griffes $15 \mu \mathrm{m}$. Longueur empodium : P1. $18 \mu \mathrm{m}$, P2 $24 \mu \mathrm{m}$, P3 $21 \mu \mathrm{m}$.

Mesures des pattes en microns $(n=1)$ :

\begin{tabular}{lccccccc}
\hline & fe & ti & tal & ta2 & ta3 & ta4 & ta5 \\
P1 & 353 & 425 & 202 & 137 & 93 & 58 & 58 \\
P2 & 418 & 410 & 173 & 115 & 82 & 47 & 51 \\
P3 & 432 & 439 & 223 & 122 & 115 & 57 & 57 \\
& LR & BV & SV & BR & & \\
P1 & 0,47 & 2,83 & 3,85 & $1,1-1,3$ & & \\
P2 & 0,42 & 3,39 & 4,79 & $1,5-1,6$ & & \\
P3 & 0,50 & 3,12 & 3,90 & $1,7-2,0$ & & \\
\hline
\end{tabular}

\section{- Abdomen}

Segments I à V de couleur brun clair, segments VI-VIII et hypopyge brun plus foncé. Tergites abdominaux avec soies peu nombreuses et espacées : seulement 11-12 soies sur les tergites I à IV.

\section{- Hypopyge (fig. 2)}

Le lobe interne du gonocoxite et la pointe anale sont très semblables à ceux de Th. gracilis (Brundin 1956, fig. 99). Pointe anale longue et sans soies; lame anale avec 6-8 macrosoies (environ 15 macrosoies chez Th. gracilis). Gonocoxite long de $10 \mu \mathrm{m}$, gonostyle triangulaire long de $55 \mu \mathrm{m}$ : le bord externe se prolonge parfois en forme d'une petite dent. $\mathrm{HR}=0,44 . \mathrm{HV}=1,14$.

Un gonostyle de forme triangulaire se rencontre chez plusieurs autres genres d'Orthocladiinae notamment chez Chaetocladius laminatus (Brundin 1947, fig. 50), Hydrobaenus conformis (Brundin 1956, fig. 40, sub. Trissocladius) et Eukiefferiella sivertseni (Aagaard 1979).

Le bord externe du gonostyle peut même se prolonger en une dent nettement plus grande comme par exemple chez Chaetocladius dentiforceps (Pinder 1978, fig. 123 A) ou Zalutschia humphriesiae (Dowling \& Murray 1978).

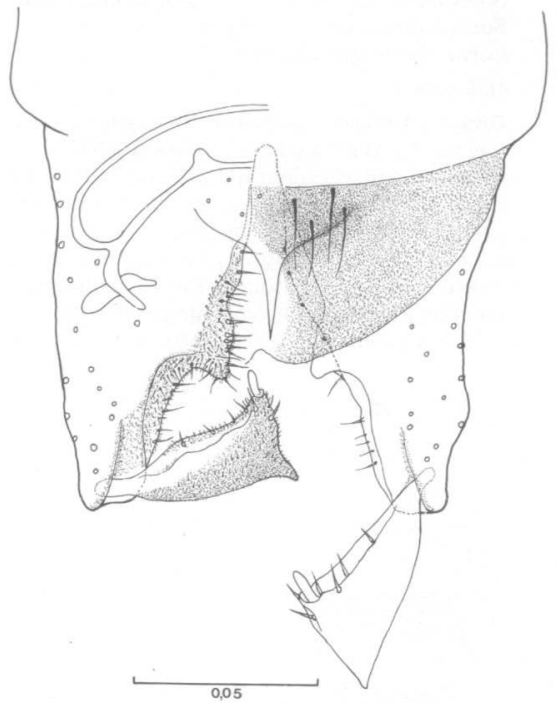

Fig.2. Thienemannia libanica n. sp. $\sigma$ : hypopyge. Echelle en $\mathrm{mm}$.

\section{Nymphe $(\mathrm{n}=3)$}

Exuvie nymphale transparente. Longueur 2,1-2,3 $\mathrm{mm} ; \mathrm{L}$ abdomen $1,4-1,5 \mathrm{~mm}$.

Les longueurs de la nymphe de $T h$. gracilis sont variables selon les auteurs: 1,8-2,0 mm (Langton 1984), $2,4 \mathrm{~mm}$ (Pyrénées: Touyre ${ }^{2}$ ), $2,7 \mathrm{~mm}$ (Potthast 1914), $3 \mathrm{~mm}$ (Thienemann 1944).

\section{- Céphalothorax}

Chaetotaxie :

Soies frontales, $\mathrm{L}=15 \mu \mathrm{m}$ (pas de tubercules). Soies antepronotales médianes, $\mathrm{L}=27 \mu \mathrm{m}, 36 \mu \mathrm{m}$. Soies précornéales, $\mathrm{L}=30 \mu \mathrm{m}, 25 \mu \mathrm{m}, 37 \mu \mathrm{m}$.

2. Récentes captures dans les Pyrénées centrales: rivière Touyre, sous-affluent de l'Ariège: station Montferrier $(670 \mathrm{~m})$ le 30.7.82: 1E \&; station Nestor $(560 \mathrm{~m})$ le 21.4 .82 : 1 No (leg. Brouquet). 
Soies dorsocentrales, $\mathrm{L}=10 \mu \mathrm{m}, 12 \mu \mathrm{m}, 12 \mu \mathrm{m}$.

Soies supraalaires, $\mathrm{L}=9 \mu \mathrm{m}$.

Corne thoracique absente.

\section{- Abdomen}

Ornementation et Chaetotaxie des tergites comme sur la fig. 3 a. Bord anal des tergites II à VIII avec une rangée transversale d'épines aussi hautes que larges sur les tergites II à IV, puis plus étroites sur les tergites V à VII. La partie comprise entre cette rangée d'épines et la suture intersegmentaire est garnie d'un chagrin continu de fines spinules légèrement ondulées avec la pointe dirigée vers l'avant. Ce chagrin est interrompu au centre du tergite VII.
Chez Th. gracilis il est interrompu au centre des tergites VI et VII (fig. $3 \mathrm{~d}$ ).

Bord oral du tergite III avec une bande transversale de très fines spinules alignées par petits grou. pes que l'on retrouve, mais progressivement interrompue dans la partie médiane, sur les tergites IV à VIII.

Tergites VII et VIII avec une plaque médiane plus ou moins circulaire d'épines deux fois plus nombreuses (45-50) sur le tergite VIII.

Cette plaque existe également sur le VI ${ }^{e}$ tergite de Th. gracilis ce qui permet de différencier aisément les deux exuvies (fig. 3 a et e).
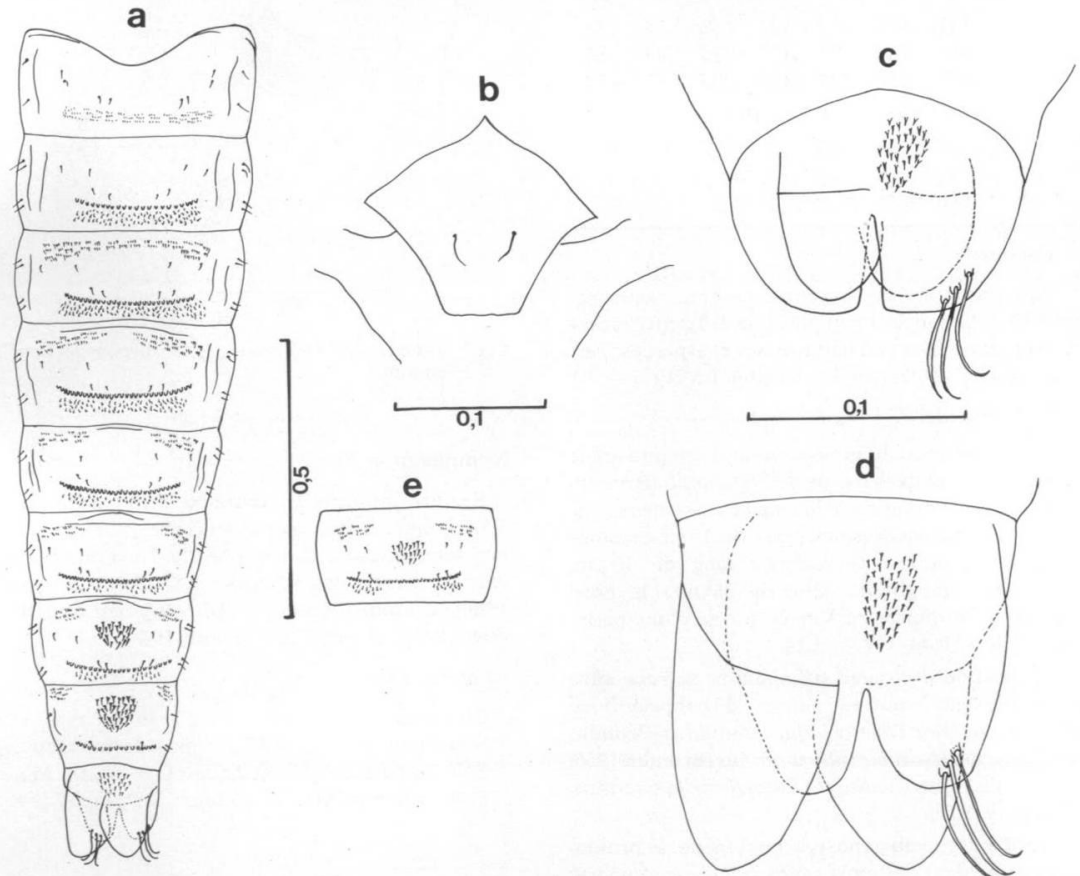

Fig. 3. Thienemannia libanica $n$. sp. Nymphe : a, abdomen - b, pièce frontale - $c$, segment anal femelle - d, segment anal mâle; Thienemannia gracilis $\mathrm{K}$ : e VIc tergite de la nymphe. Echelle en $\mathrm{mm}$. 
Segment anal ( $f$ ig. $3 \mathrm{c}$ et d) avec une zone de spinules centrales moins fortes que sur les tergites VII et VIII. Les 3 soies apicales légèrement recourbées et longues de 51 à $57 \mu \mathrm{m}$ atteignent presque la moitié du lobe anal $(120 \mu \mathrm{m})$.

LAR $\sigma=2,14$; LAR 9 Th. gracilis (Touyre/Pyrénées) $=2,12$.

\section{Larve inconnue}

Récemment, Cranston \& al. (1983) ont décrit deux formes larvaires paléarctiques appartenant au genre Thienemannia: Thienemannia gracilis et Thienemannia sp.

\section{Ecologie et répartition}

Thienemannia gracilis est une espèce crénophile ou hygropétrique (Spärck 1922, Thienemann 1937). Elle est également signalée dans les mousses des rivières de montagne à différentes altitudes notamment dans les Alpes à $825 \mathrm{~m}$ (Thienemann 1936), dans les Carpathes (Albu 1966), dans la Sierra Nevada à $2400 \mathrm{~m}$ (Laville 1970), dans les Pyrénées au-dessous de $400 \mathrm{~m}$ (Laville 1980) et dernièrement entre 670 et $560 \mathrm{~m}$ dans un sous-affluent de l'Ariège' ${ }^{2}$. Elle a été trouvée dans les Mittelgebirge occidentales, notamment dans des rivières du Jura (Verneaux 1968, Verneaux et Vergon 1974) et dans les Mittelgebirge centrales comme dans l'Epirhithral de la Fulda (Lehmann 1971) ou dans les faciès hygropétriques de Sauerland (Thienemann 1954). Elle est connue des rivières de plaine d'Europe centrale (Caspers 1980) et orientale (Pankratova 1970).

Brundin (1956) l'a signalée de Suède, Belgique, Angleterre, Islande.

Jusqu'ici, cette espèce n'avait jamais été trouvée en grand nombre; récemment, Caspers (1983) en signale d'abondantes récoltes ( 1082 imagos) près de Lunz, dans la rivière Schreierbach $(700 \mathrm{~m})$ dans les Alpes autrichiennes. Th. gracilis parait donc assez largement répartie du Crénal au Rhithral de nombreux cours d'eau d'Europe occidentale.

Nous n'avons que peu de données écologiques con. cernant Thienemannia libanica $\mathrm{n}$. sp. récoltée à une seule station proche de la localité de Chlifa (1 100-1 $200 \mathrm{~m}$ ) dans la rivière à régime torrentiel Yammouné. Cette station de moyenne montagne sur le versant Est du Mont Liban et assimilèe à un métarhithral, présente un substrat en grande partie rocheux couvert d'un tapis très dense de bryophytes et d'hydrures. Le courant est très rapide $(2 \mathrm{~m} / \mathrm{s})$ et la température de l'eau varie entre $8^{\circ} 5$ (min.) et $12^{\circ} \mathrm{C}$ (max.). Ces conditions sont comparables à celles de nombreuses stations où $T h$ gracilis a été trouvée. Dans cette station, 15 espèces de Chironomides ont été recensées. Parmi elles, 2 Diamesinae Boreoheptagia legeri et Diamesa kasymovi et les Orthocladinae Corynoneura lobata, Eukiefferiella coerulescens, E. dittmari, E. fuldensis, E. lobifera, E. pseudomontana, Orthocladius thienemanni, $O$. frigidus.

Ces Orthocladiinae assez psychrothermes ont été souvent signalés dans les massifs montagneux : ils colonisent notamment la rivière Lot entre $1295 \mathrm{~m}$ et $443 \mathrm{~m}$ soit, du Crénal au Métarhithral (Laville 1981).

\section{Travaux cités}

Albu (P.). 1966. - Verzeichnis der bis jetzt aus Rumänien bekannten Chironomiden. Gevässer Abwässer, 41/42: 145-146.

Aagaard (K.). 1979. - Eukiefferiella sivertseni n. sp. from Norway. (Diptera : Chironomidae), Ent. scand, Suppl, 10:95-97.

Brindin (Lr). 1947. - Zur Kenntnis der schwedischen Chi ronomiden. Ark. Zool., $39: 1-95$.

Brundin (L.). 1956. - Zur Systematik der Orthocladinae (Dipt, Chi ronomidae). Rep. Inst. freshwat. Res, Drotningholm, $37: 5185$

Caspers (N.). 1980. - Die Emergenz eines kleinen Waldbaches bei Bonn. Decheniana-Beihefle (Bonn), $23: 1-175$.

Caspers (N.). 1983. - Chironomiden-Emergenz zweier Lunzer Bäche, 1972. Arch. Hydrobiol, Suppl. 65:485-549.

Cranston (P.S.), Oliver (D.R.) \& Saether (O.A.). 1983. - The larvae of Orthocladiinae (Diptera : Chironomidae) of the Holarctic region. Keys and diagnoses. Ent. scand., suppl. 19 : 149-291.

Dowling (C.) \& Murray (D.A.). 1978. - Zalutschia humphriesiae sp. n. a new species of Orthocladinae (Diptera, Chironomidae) from Ireland. Acta Univ. Carolinae-Biologica 1980, $1 / 2$ : 49-58.

Langton (P.H.). 1984 _ A key to pupal exuviae of British Chironomidae. P.H. Langton, edit, 324 p.

Laville (H.). 1970. - Some Chironomidae (excl. Diamesinae) from southern Spain (Insecta, Diptera). Steenstrupia, I : 21-23.

Laville (H.). 1980. - Inventaire 1980 des Chironomides (Diptera) connus des Pyré nées. Annis Limnul., 16 (3): 211-223.

Laville (H.) 1981 . - Récoltes d'exuvies nymphales de Chironomides (Diptera) dans le Haut-Lot, de Ja source (1 $295 \mathrm{~m}$ ) au confluent de la Truyère (223 m). Annls Limmol, 17 (3) : 255-289.

Lehmann (J.). 1971. - Die Chironomiden der Fulda (Systematische, ökologische und faunistische Untersuchungen). Arch. Hydrobiol., suppl. 37 : $466-555$

Moubayed (Z.) \& Laville (H.). 1983. — Les Chironomidés (Diptera) du Liban. I. Premier inventaire faunistique. Annls Limnol., 19 (3) : 219.228.

Pankratova (V. Ya). 1970. - Larvae and pupae of midges of the subfamily Orthocladiinae (Diptera, Chironomidae $=$ Tendipedidae of the U.S.S.R. fauna (en russe). Itd. Nauka, Leningr., 344 p.

Pinder (L.C.V.). 1978. - A key to the adult males of British Chironomidae. Vol. 1, the Key : vol. 12, Illustrations of the hypopygia. Freshwat. Biol. Assoc. Scient. Publ. $37: 169$ p. +189 fig.

Potthast (A.). 1914 - Uber die Metamorphose der OrthocladiusGruppe. Ein Beitrag zur Kenntnis der Chironomiden. Arch. Hydrobiol, suppl. 2: 243-376.

Saether (O.A.). 1980. - Glossary of chironomid morphology terminology (Diptera : Chironomidae). Ent. scand., suppl. 14:1-51. 
Spärck (R.). 1922. - Beiträge zur Kenntnis der Chironomidenmetamorphose I-IV. Ent Meddr, 14:32-109.

Thienemann (A.). 1936. - Alpine Chironomiden (Ergebnisse von Untersuchungen in der Gegend von Garmisch-Partenkirchen, Oberbayern). Arch. Hydrobiol, $30: 167-262$.

Thienemann (A.). 1937. - Chironomiden-Metarnorphosen (Diptera) XV. Mitt ent Ges. Halle, $15: 22-36$.

Thienemann (A.). 1944. - Bestimmungstabellen für die bis jetzt bekannten Larven und Puppen der Orthocladiinen (Diptera, Chironomidae). Arch. Hydrobiot, $39: 551-664$.
Thienemann (A.). 1954 - Chironomus. Leben, Verbreitung und wirtschafliche Bedeutung der Chironomiden. Binnengewässer $20: 834 \mathrm{p}$.

Verneaux (J.). 1968. - Contribution à l'étude d'une petite rivière de plaine, les Doulonnes (Jura). Référence particulière aux Diptères Chironomides. Remarques ecologiques. Annls scient. Univ. Besançon, $3^{\text {e }}$ sér., $4: 29.40$.

Verneaux (J.) \& Vergon (J.P.). 1974. - Faune dulçaquicole de Franche-Cornté. Sixième partie : les Diptères Chironomides. Annls scient. Univ. Besançon, 3e sér., 11 : 179-198. 